\title{
Beer Potomania: A Challenging Case of Hyponatremia
}

\author{
Hind Rafei ${ }^{a}$, Raza Yunus ${ }^{\mathrm{a}}$, Parvinder Khurana ${ }^{\mathrm{a}, \mathrm{b}}$
}

\begin{abstract}
Beer potomania is a syndrome of hyponatremia associated with excessive beer drinking. Little or no salt content of beer results in marked reduction in the solute load to the kidney. This leads to impaired water clearance and dilutional hyponatremia. A 66-year-old man with history of alcoholism and alcoholic cardiomyopathy presented to the emergency room with tremors of his upper and lower extremities. He had a significant history of alcohol consumption, usually drinking 4 - 5 cans of beer per night for the past 34 years. In addition, he had consumed a fifth of a vodka bottle the day before presentation. He had a pattern of often skipping meals though was compliant with both his diuretics medications: furosemide $40 \mathrm{mg}$ once daily and spironolactone 25 mg daily. On physical exam, he was euvolemic. Neurological exam revealed resting tremors of both his hands. Labs were remarkable for plasma sodium of 122 , brain natriuretic peptide of 474 , serum osmolality of 268, urine osmolality of 223, and urine sodium of 20. Patient was assessed to have moderate euvolemic hypotonic hyponatremia. The combination of euvolemic hyponatremia with history of excessive beer drinking made beer potomania very likely. His urine osmolality and urine sodium, however, were higher than expected in beer potomania. These could be explained by the two diuretics that the patient was taking. Patient was managed with fluid restriction, appropriate nutritional and sodium intake and withholding of his diuretics. Plasma sodium slowly corrected to 130 over the course of 3 days. This case illustrates the condition beer potomania, an infrequent cause of hyponatremia. Findings in hyponatremia do not always point in one direction, especially with the concomitant use of diuretics. Recognition of beer potomania is critical as it is associated with serious neurologic sequelae that should be part of counseling against alcohol abuse.
\end{abstract}

Keywords: Beer potomania; Hyponatremia; Diuretics

\section{Introduction}

A syndrome of hyponatremia associated with excessive beer drinking was first recognized by Demanet et al in 1971 and has

Manuscript accepted for publication June 21, 2016

aDepartment of Internal Medicine, George Washington University Hospital, 2150 Pennsylvania Ave NW, Washington, DC 20037, USA

${ }^{b}$ Corresponding Author: Parvindor Khurana, Department of Internal Medicine, George Washington University Hospital, 2150 Pennsylvania Ave NW, Washington, DC 20037, USA. Email: pkhurana@mfa.gwu.edu

doi: http://dx.doi.org/10.14740/jem350e been referred to as beer potomania [1]. The term beer potomania is used to describe a patient who presents with hyponatremia in conjunction with low daily solute intake and excessive beer drinking. Little or no salt content of beer, and suppression of protein breakdown by the carbohydrate and alcohol content of beer, result in marked reduction in the solute load to the kidney [2]. The low solute load leads to impaired water clearance and consequently dilutional hyponatremia [3]. Beer potomania is difficult to recognize especially in the presence of other factors commonly affecting sodium excretion such as diuretics. This article presents a case report of a 66-year-old alcoholic man who presented with moderate euvolemic hyponatremia due to beer potomania where the diagnosis was challenging in the setting of diuretic intake.

\section{Case Report}

A 66-year-old man with history of alcoholism and alcoholic cardiomyopathy presented to the emergency room with tremors of his upper and lower extremities. History dated back to 6 days prior to presentation when he developed tremors of all his four extremities and was unable to walk. He tripped at home but denied loss of consciousness or head trauma. He had a significant history of alcohol consumption, usually drinking 4 - 5 cans of beer per night for the past 34 years. In addition, he had consumed a fifth of a vodka bottle the day before presentation. He had a pattern of often skipping meals though was compliant with both his diuretics medications: furosemide $40 \mathrm{mg}$ once daily and spironolactone $25 \mathrm{mg}$ once daily. He was not on any antipsychotic or antidepressant drugs. His history was negative for seizures, confusion or somnolence. He denied diarrhea, vomiting, cold intolerance, shortness of breath, swelling, or excessive water intake. He denied cough, chest pain, hemoptysis, weight loss, fevers, or night sweats. On physical exam, he was afebrile, hypertensive to $165 / 75$, with a heart rate of 67 , respiratory rate of 18 , and an oxygen saturation of $100 \%$. He was awake, cooperative and in no acute distress. He was euvolemic with no jugular venous distention, lower extremity edema or pulmonary crackles. Neurological exam revealed resting tremors of both his hands with no asterixis.

His laboratory workup included a complete blood count that revealed a white blood count of $4.8 \times 10^{3} / \mu \mathrm{L}$, hemoglobin of $10.8 \mathrm{~g} / \mathrm{dL}$, hematocrit of $32.1 \%$, and a platelet count of 156 $\times 10^{3} / \mu \mathrm{L}$. His basic chemistries on admission showed hyponatremia with a sodium level down to $122 \mathrm{mmol} / \mathrm{L}$, a potassium level of $5 \mathrm{mmol} / \mathrm{L}$, chloride of $88 \mathrm{mmol} / \mathrm{L}$, bicarbonate of 28 $\mathrm{mmol} / \mathrm{L}$, blood urea nitrogen of $8 \mathrm{mg} / \mathrm{dL}$ and a creatinine of 1 $\mathrm{mg} / \mathrm{dL}$. Blood glucose on admission was $114 \mathrm{mg} / \mathrm{dL}$. Mineral panel showed calcium of $8.6 \mathrm{mg} / \mathrm{dL}$, phosphate of $3.3 \mathrm{mg} / \mathrm{dL}$, 


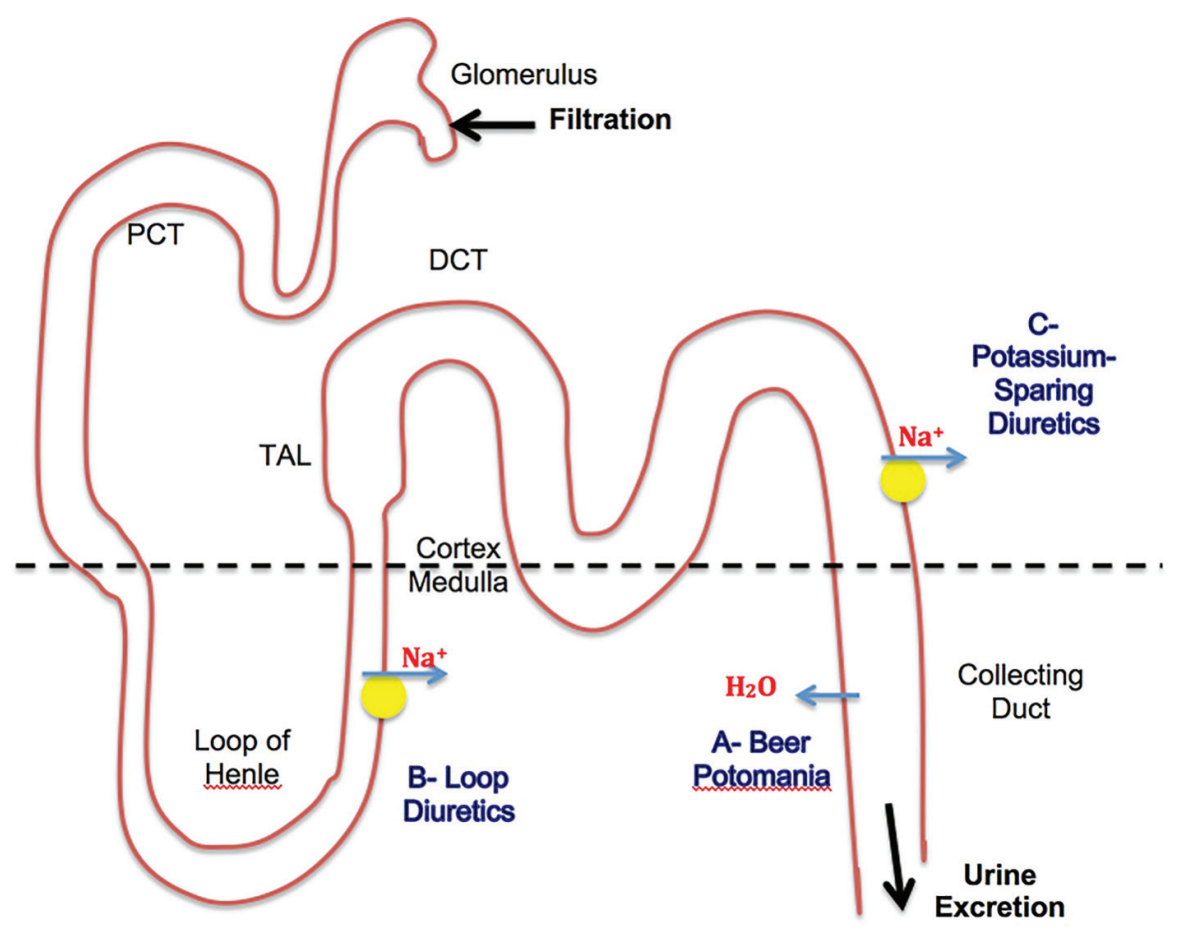

Figure 1. Mechanisms involved in patient's presentation. (A) Beer has low sodium and protein + poor diet $=$ low total body solute. Low body solute leads to hypotonic hyponatremia. ADH is suppressed. Water is excreted in urine $\rightarrow$ leads to low urine osmolality (usually < 100). Solute is less in urine $\rightarrow$ leads to low urine $\mathrm{Na}$ (usually $<20$ ). (B) Loop diuretics lead to increase excretion of $\mathrm{Na}$ $\rightarrow$ also leads to increase in urine osmolality. (C) K sparing diuretics lead to increase excretion of $\mathrm{Na} \rightarrow$ also leads to increase in urine osmolality. $\mathrm{B}+\mathrm{C}$ leads to increase in urine $\mathrm{Na}$ to 20 and urine osmolality to 223 in our case.

and magnesium of $1.7 \mathrm{mg} / \mathrm{dL}$. Liver function tests were positive for 2:1 ratio of AST to ALT with AST of $61 \mathrm{IU} / \mathrm{L}$ and ALT of $30 \mathrm{IU} / \mathrm{L}$. Otherwise, total protein was $5.7 \mathrm{~g} / \mathrm{dL}$, albumin was $3.6 \mathrm{~g} / \mathrm{dL}$, alkaline phosphatase was $79 \mathrm{mU} / \mathrm{mL}$, total bilirubin was $1.4 \mathrm{mg} / \mathrm{dL}$, direct bilirubin was $0.2 \mathrm{mg} / \mathrm{dL}$ and INR was 1. Brain natriuretic peptide (BNP) was $474 \mathrm{pg} / \mathrm{mL}$ compared to $4,000 \mathrm{pg} / \mathrm{mL}$ on a previous admission for congestive heart failure exacerbation. Thyroid stimulating hormone (TSH) was $1.12 \mu \mathrm{IU} / \mathrm{mL}$. Serum osmolality was $268 \mathrm{mOsm} / \mathrm{kg} \mathrm{H}_{2} \mathrm{O}$ while urine osmolality was $223 \mathrm{mOsm} / \mathrm{kg} \mathrm{H}_{2} \mathrm{O}$ with urine sodium of $20 \mathrm{mmol} / \mathrm{L}$. A chest X-ray was also done and was normal.

Patient was admitted to the hospital for management of hyponatremia. Based on the history of excessive beer drinking with recent binge drinking as well as the physical exam and laboratory findings suggestive of hypotonic euvolemic hyponatremia, patient was diagnosed with beer potomania especially that other known causes of hyponatremia were excluded. Management consisted of fluid restriction, adequate nutritional supplementation and withholding of the two diuretics. Intravenous fluids were not administered, as patient was not acutely symptomatic. Plasma sodium was frequently monitored. It slowly corrected up to 130 over the course of 3 days.

\section{Discussion}

Based on the low serum osmolality of $268 \mathrm{mOsm} / \mathrm{kg} \mathrm{H}_{2} \mathrm{O}$, hyponatremia was categorized as hypotonic hyponatremia [4]. It was further considered moderate due to a level between 120 and $130 \mathrm{mmol} / \mathrm{L}$ [5]. The fact that he presented 6 days after the onset of symptoms and that he lacked major neurologic symptoms such as seizures, confusion, somnolence, etc. made chronic hyponatremia ( $>48 \mathrm{~h}$ ) more likely [6]. Congestive heart failure exacerbation as the etiology of hyponatremia was excluded in this case due to many factors. Patient lacked attributable signs and symptoms such as shortness of breath, orthopnea, volume overload, cardiopulmonary decompensation on chest X-ray, elevated BNP, etc. Furthermore, TSH was normal which excluded hypothyroidism. Psychogenic polydipsia was unlikely as patient denied excessive water intake and urine osmolality was not less than $100 \mathrm{mOsm} / \mathrm{kg} \mathrm{H} \mathrm{H}_{2} \mathrm{O}$. History, physical exam and workup made SIADH also an unlikely explanation. Chest $\mathrm{X}$-ray was negative for a pulmonary process. Malignancy was not a concern at that point as patient denied systemic symptoms of weight loss, fevers, night sweats and was up-to-date with age-specific screening. History was negative as well for intake of antipsychotics, antidepressants or other drugs that can lead to SIADH and no intracranial process was evident from history or physical exam.

The history of excessive beer drinking with recent binge drinking made beer potomania a very likely explanation of patient's euvolemic hyponatremia. The criteria used to diagnose beer potomania syndrome included a history of binge beer drinking and poor dietary intake along with decreased serum sodium levels in the absence of other known causes of hyponatremia $[7,8]$. While beer potomania is usually characterized by 
low urine sodium and urine osmolality [9], the urine osmolality and urine sodium were slightly higher than expected. These findings could be explained by the two diuretics patient was taking. Both furosemide and spironolactone can cause excretion of sodium in urine, which leads to a higher urine sodium and osmolality [10]. In a review of 22 cases of beer potomania, Sanghvi et al showed that hyposthenuria on admission was not a consistent finding in beer drinkers with hyponatremia. They concluded that recognizing this finding prevents the erroneous dismissal of beer potomania [11]. Figure 1 illustrates the pathophysiology of hyponatremia in this particular patient. Patient's presentation could not be explained by excessive diuresis from furosemide and spironolactone alone due to many factors. Patient lacked symptoms of hypovolemia. Furthermore, urine sodium in diuretic-induced hyponatremia is usually higher than in our patient (usually above $40 \mathrm{mmol} / \mathrm{L}$ ) with associated higher urine osmolality (above $450 \mathrm{mOsm} / \mathrm{kg} \mathrm{H}_{2} \mathrm{O}$ ) [12].

Beer potomania is a well-known cause of hyponatremia. It is mainly explained by water intake that exceeds excretory capacity [11]. Free water clearance is dependent on solute excretion and urinary diluting capacity [13]. The osmolar load to the kidney is caused both by urea generation from proteins as well as dietary sodium and potassium intake. Under normal conditions, typical osmole excretion is approximately $600-900$ $\mathrm{mOsm} /$ day. While the maximum urinary dilution capability is $50 \mathrm{mOsm} / \mathrm{L}$, a large amount of water $(>20 \mathrm{~L})$ must be ingested before overwhelming the urinary dilution capacity, as seen is psychogenic polydipsia. The low solute content of alcohol as well as prevention of protein breakdown (urea generation) by the carbohydrate (high calorie) content of beer in conjunction with poor dietary supplementation of sodium and potassium as seen in many alcoholics, all together, leads to a decrease in solute excretion. Consequently, the ability to excrete free water becomes limited. Because the obligatory solute loss in a day is approximately $250 \mathrm{mOsm}$ in these patients, with a urinary dilution capability of $50 \mathrm{mOsm} / \mathrm{L}$, water intake greater than $5 \mathrm{~L}$ (or 14 cans of beer) results in hyponatremia [7]. As a result, antidiuretic hormone (ADH) levels are expected to be suppressed in patients with beer potomania due to excess of water [14]. The low ADH levels limit free-water reuptake in the collecting tubules of the kidney and lead to brisk diuresis when solute is presented. Water diuresis can produce large increases in serum sodium levels in a short period, which in turn predisposes to the negative consequences of rapid correction of hyponatremia. Sequelae include in extreme cases pontine and extrapontine myelinolysis known as osmotic demyelination syndrome (ODS) due to the osmotic stress on oligodendrocytes that occurs with rapid correction of hyponatremia [15]. Thus, management of hyponatremia in beer potomania might be difficult.

In their review of beer potomania cases, Sanghvi et al [11] recommended that treatment goals for patients presenting with beer potomania should be limited to a serum sodium increase of less than $10 \mathrm{mmol} / \mathrm{L}$ in the first $24 \mathrm{~h}$ and less than $18 \mathrm{mmol} / \mathrm{L}$ in the first $48 \mathrm{~h}$. If the patient is asymptomatic, fluid restriction and patient's monitoring is the safest approach. If the desired goal is exceeded, D5W infusion might be started to match urine output. The rate of infusion can be adjusted based on sodium levels monitored every $2 \mathrm{~h}$.

\section{Conclusion}

Beer potomania is an infrequent cause of hyponatremia caused by excessive alcohol intake and poor diet. Findings in hyponatremia do not always point in one direction, especially with the concomitant use of diuretics. Thus, understanding of pathophysiology can be useful in interpreting lab results. Recognition of beer potomania as a separate entity of hyponatremia is critical, as management should be tailored to the specific pathophysiologic state of this condition. If unrecognized, beer potomania can lead to serious neurologic sequelae. Patient education and alcohol-related counseling are essential to prevent recurrence.

\section{Disclosure}

Authors have no financial or non-financial relationships to disclose.

\section{References}

1. Demanet JC, Bonnyns M, Bleiberg H, Stevens-Rocmans C. Coma due to water intoxication in beer drinkers. Lancet. $1971 ; 2(7734): 1115-1117$.

2. Imam TH. Taking alcohol with a (large) pinch of salt: Understanding the osmoles in "beer potomania" and "starvation potomania". Indian J Nephrol. 2014;24(4):203-205.

3. Harrow AS. Beer potomania syndrome in an alcoholic. Va Med. 1989;116(6):270-271.

4. Sterns RH. Diagnostic evaluation of adults with hyponatremia. In: Emmet M, Forman JP. (Eds.) UptoDate. 2015. Available from http:/www.uptodate.com/contents/diagnostic-evaluation-of-adults-with-hyponatremia.

5. Dasta J, Waikar SS, Xie L, Boklage S, Baser O, Chiodo J, 3rd, Badawi O. Patterns of treatment and correction of hyponatremia in intensive care unit patients. J Crit Care. 2015;30(5):1072-1079.

6. Goh KP. Management of hyponatremia. Am Fam Physician. 2004;69(10):2387-2394.

7. Fenves AZ, Thomas S, Knochel JP. Beer potomania: two cases and review of the literature. Clin Nephrol. 1996;45(1):61-64.

8. Hilden T, Svendsen TL. Electrolyte disturbances in beer drinkers. A specific "hypo-osmolality syndrome". Lancet. 1975;2(7928):245-246.

9. Liamis GL, Milionis HJ, Rizos EC, Siamopoulos KC, Elisaf MS. Mechanisms of hyponatraemia in alcohol patients. Alcohol Alcohol. 2000;35(6):612-616.

10. Bernstein PL, Ellison DH. Diuretics and salt transport along the nephron. Semin Nephrol. 2011;31(6):475-482.

11. Sanghvi SR, Kellerman PS, Nanovic L. Beer potomania: an unusual cause of hyponatremia at high risk of complications from rapid correction. Am J Kidney Dis. 2007;50(4):673-680. 
12. Milionis HJ, Liamis GL, Elisaf MS. The hyponatremic patient: a systematic approach to laboratory diagnosis. CMAJ. 2002;166(8):1056-1062.

13. Thaler SM, Teitelbaum I, Berl T. "Beer potomania" in non-beer drinkers: effect of low dietary solute intake. Am J Kidney Dis. 1998;31(6):1028-1031.
14. Kamel KS, Halperin ML. The importance of distal delivery of filtrate and residual water permeability in the pathophysiology of hyponatremia. Nephrol Dial Transplant. 2012;27(3):872-875.

15. Laureno R, Karp BI. Myelinolysis after correction of hyponatremia. Ann Intern Med. 1997;126(1):57-62. 